\title{
Laurencia viridis sp. nov. (Ceramiales, Rhodomelaceae) from the Macaronesian Archipelagos
}

\author{
M. C. Gil-Rodríguez and R. Haroun \\ Department of Vegetal Biology (Botany), University of La Laguna, 38271 La Laguna, Tenerife, Canary Islands, \\ Spain
}

(Accepted 14 April 1992)

\begin{abstract}
Laurencia viridis sp. nov. (Ceramiales, Rhodomelaceae) is described from specimens collected around the Canary Islands and herbarium examples from Azores Islands, Madeira Islands, Salvage Islands and Cape Verde Islands. Vegetative fronds, sporophytes and gametophytes (male and female) arise from stoloniferous axes.

Characteristics of this new species include the following: a distinct green colour of both vegetative and fertile plants, with pinkish apical regions; presence of secondary pit-connections between adjacent cortical cells; terete branches radially arranged; spermatangial receptacle of determinate position, cup-like and with a clear central axis; tetrasporangia in parallel abaxial rows. This species is placed in the genus Laurencia Lamouroux, subgenus Laurencia Saito, section Laurencia Saito. Ecological data are presented.
\end{abstract}

We compare the $L$. viridis from the Macaronesian Archipelagos with the green species of Laurencia from other parts of the world.

\section{Introduction}

The genus Laurencia Lamouroux (Rhodomelaceae) includes about 140 species (McDermid 1989) of small to medium-sized red algae, distributed worldwide except in the Arctic and Antarctic. Members of this genus are abundant in warm waters, however, and often constitutes a conspicuous part of subtropical and tropical floras (Saito 1969, McDermid 1988, Vandermeulen et al. 1990).

Several species are present in the Macaronesian Archipelago, where they are a conspicuous component of the intertidal vegetation. Thirteen species were reported from Canary Islands area by Gil-Rodríguez and Haroun (1992) and fifteen species in a further publication by Gil-Rodríguez (1992). One of these species, for which fertile material was lacking, was designated as Laurencia sp. 1 (grex obtusa). Fertile specimens have subsequently been collected in various localities around Tenerife, La Palma, Hierro, Lanzarote, Montaña Clara and herbarium specimens ex- amined from Azores (Santa María), Madeira Islands (Porto Santo), Salvage Islands (Fora and Salvage Pequeña) and Cape Verde Islands (Sao Vicente, Santiago, Branco and Sal). The plant is now described as a new species, Laurencia viridis.

\section{Material and Methods}

The description of this new species is based chiefly on plants collected intertidally in the Canary Islands and herbarium specimens (L!) from Azores Islands, Madeira Islands, Salvage Islands and Cape Verde Islands. Anatomical studies were made on both fresh specimens and plants fixed in $4 \%$ formalin in sea water. Slide preparations were mounted in $80 \%$ corn syrup (Karo ${ }^{\circledR}$ ) after staining for $5-10$ min with $1 \%$ aqueous aniline blue (Womersley 1984). Dried material of sterile plants, sporophytes and gametophytes is deposited at TFC Phyc. (Herbario de la Universidad de La Laguna, Departamento de Biología Vegetal, Botánica, Tenerife, Islas Canarias): 
Tenerife: Punta del Hidalgo (10-1980, TFC Phyc. 732; 10-06-1986, TFC Phyc. 4289; 16-06-1986, TFC Phyc. 4288; 20-06-1986, TFC Phyc. 7177; 30-06-1986, TFC Phyc. 7203; 22-10-1988, TFC Phyc. 7183; 5-11-1988, TFC Phyc. 7207; 12-12-1988, TFC Phyc. 7188; $12-$ 01-1989, TFC Phyc. 7202; 22-01-1989, TFC Phyc. 7189; 22-02-1989, TFC Phyc. 7206; 7-05-1989, TFC Phyc. 7200; 6-06-1989, TFC Phyc. 7204; 13-06-1989, TFC Phyc. 7185; 3-03-1990, TFC Phyc. 7174; 3-051990, TFC Phyc. 7182). Bajamar (3-06-1990, TFC Phyc. 7179). Las Caletillas (08-1979, TFC Phyc. 1789; 5-07-1985, TFC Phyc. 7208). Güimar-Playa del Socorro (28-04-1983, TFC Phyc. 175; 27-01-1987, TFC Phyc. 7209). Porís de Abona (03-1989, TFC Phyc. 7175). Tajao (11-06-1986, TFC Phyc. 4293; 10-031989, TFC Phyc. 7190; 12-04-1989, TFC Phyc. 7201). El Palmar (8-02-1989, TFC Phyc. 7181). Callao Salvaje (22-10-1979, TFC Phyc. 788; 3-05-1983, TFC Phyc. 7186; 13-05-1983, TFC Phyc. 174; Paraíso Floral (5-02-1989, TFC Phyc. 7180; 30-01-1990, TFC Phyc. 7173).

La Palma: Fajana de Barlovento (15-06-1983, TFC Phyc. 3044).

Hierro: El Verodal (3-04-1989, TFC Phyc. 7184). Tacorón (6-04-1989, TFC Phyc. 7187). La Restinga (504-1989, TFC Phyc. 7205).

Lanzarote: Timanfaya-Playa del Cochino (11-081987, TFC Phyc. 5204). Montaña Clara (31-03-1983, TFC Phyc. 7178).

Additional material examined from Rijksherbarium Leiden (L).

Azores Islands: Cancap V (4805), STA 5K03. S. coast Santa Maria (29-5-1981) 987.276/051 (as L. obtusa var. pyramidata).

Madeira Islands: Cancap IV (4135), STA 4K27. Porto Santo (10-6-1980) 987.187/309 (as L. obtusa (Hudson) Lamouroux).

Salvage Islands: Cancap IV (3396). Isle do Fora (265-1980) 987.251/240 (as L. obtusa (Hudson) Lamouroux); Cancap IV (3373), STA 4DO7. Salvage Pequeña (26-5-1980) $987.251 / 363$ (as L. obtusa (Hudson) Lamouroux).

Canary Islands: Cancap IV (3619), STA 4D12. La Palma (30/31-5-1980) 987.252/890 (as Laurencia sp.).

Cape Verde Islands: Cancap VI (7428), STA 6D11. Sao Vicente (Baia San Pedro, 20-6-1980) 988.111/243 (as Laurencia sp.); Cancap VI (6571), STA 6K07. Santiago (Baia de Santa Clara, 6-6-1982) 987.326/270 (as L. obtusa); Cancap VI (6564), ibis, 987.326/228 (as Laurencia sp.); Cancap VII (9712), STA 7K24. I.
Branco (5-9-1986) 987.170/282 (as Laurencia sp.); Cancap VII (9689), ibid, 987.170/439 (as Laurencia sp.); Cancap VII (9672), ibid, 987.170/175 (as Laurencia sp.); Cancap VII (9292), STA 7D08. Sal (29-81986) $987.170 / 216$ (as L. obtusa); Cancap VII (9291), ibid, 987.170/218 (as L. obtusa); Cancap VII (9189), STA 7K16. I. do Sal Rei (28-8-1986) 987.170/319 (as Laurencia sp.); Cancap VII (8848), STA 7K10. Santiago (21-8-1986) 987.117/719 (as L. obtusa); Cancap VII (8847), ibid, 987.118/703 (female plant) (as $L$. obtusa); Cancap VI (6432), STA 6K06. Santiago (4/ 5/6/11-6-1982) 987.326/397 (as L. obtusa); Cancap VI (6434), ibid, 987.326/365 (as Laurencia sp.).

\section{Results \\ Diagnosis}

Laurencia viridis Gil-Rodriguez et Haroun sp. nov.

Thallus annus erectus, cartilagineus, papyro non adhaerens, colore viridi propio. Axes principales erecti, $2-(4-8)-15 \mathrm{~cm}$ alti, rami clavati, colori viridi, apicibus rosaceis; aliqui axes surgunt ab axibus qui substrato per rhizoidea se adhaerent. Ramificatio alternata vel irregularis et generaliter tantum in partibus superioribus, in inferioribus sine ramificatione.

Spermatangia cupuliformia, in quoque ramo varia, 1,5-2-(3) mm longa et 1-1,5 mm lata. Cystocarpi urceolati sessiles, $1,(3-n)$ in quoque ramo, 11,5(2) $\mathrm{mm}$ longi $\times 0,5-1-(1,5) \mathrm{mm}$ late. Tetrasporangia disposita parallele axi ramli, abaxialia.

Habit: frequens in locis valde expositis fluctibus.

Holotype. Tetrasporangial plants collected at Punta Hidalgo-Baja Negra (northern part of Tenerife, Canary Islands) in the lower intertidal zone mixed with other algae, 8 May 1989, leg. M. C. Gil-Rodríguez, TFC Phyc. 7176. (Fig. 1A)

Isotypes. Tetrasporangial plants collected at the same locality as the holotype, 8 May 1989 (BM, P, L, C, SAP, MA, UC and TFC Phyc.).

Etymology: The specific name Laurencia viridis draws attention to the distinctive greenish colour of this alga.

Thallus annual, erect, cartilaginous, not adhering to paper, dark green. Erect axes $2-(4-8)-15 \mathrm{~cm}$ high; arising from stoloniferous axes that are attached to substrate by rhizoids. Branches are clavate and green but with punkish tips. Branching is alternate or helicoid and occurs only in the upper $2 / 3$ of the thallus.

Spermatangia ovoid, produced on repeatedly branched trichoblasts 1,5-2-(3) mm high $\times 1-1,5$ 

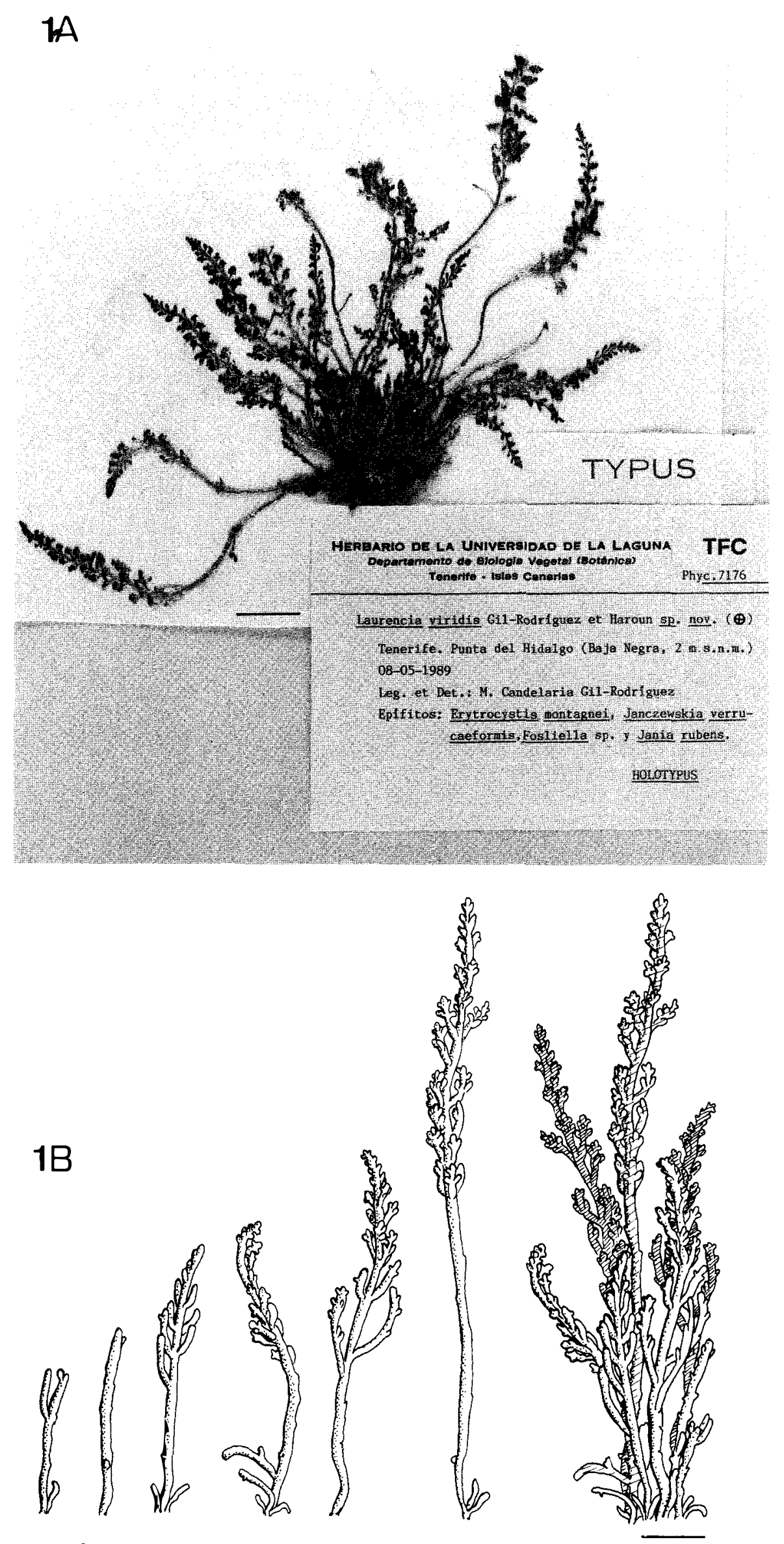

Fig. $1 \mathrm{~A}-\mathrm{B}$. Laurencia viridis $s p$. nov.

1A.: Holotypus (Scale bar $=2 \mathrm{~cm})$. 1B.: General morphology $($ Scale bar $=1.5 \mathrm{~cm})$. 
mm wide, arising from cup-like depressions at the apex of branches. Cystocarps urceolate, sessile, $1-(3-\mathrm{n})$ per branch; 0,5-1-(1,5) $\mathrm{mm}$ wide $\times 1-1,5(2) \mathrm{mm}$ high. Tetrasporangia arranged in rows parallel to the axis of the branch, abaxial.

\section{Vegetative structure}

The plant consists of annual, erect (Fig. 1B), cartilaginous, dark green, thalli arising from stoloniferous axes that are attached to the substrate by mean of
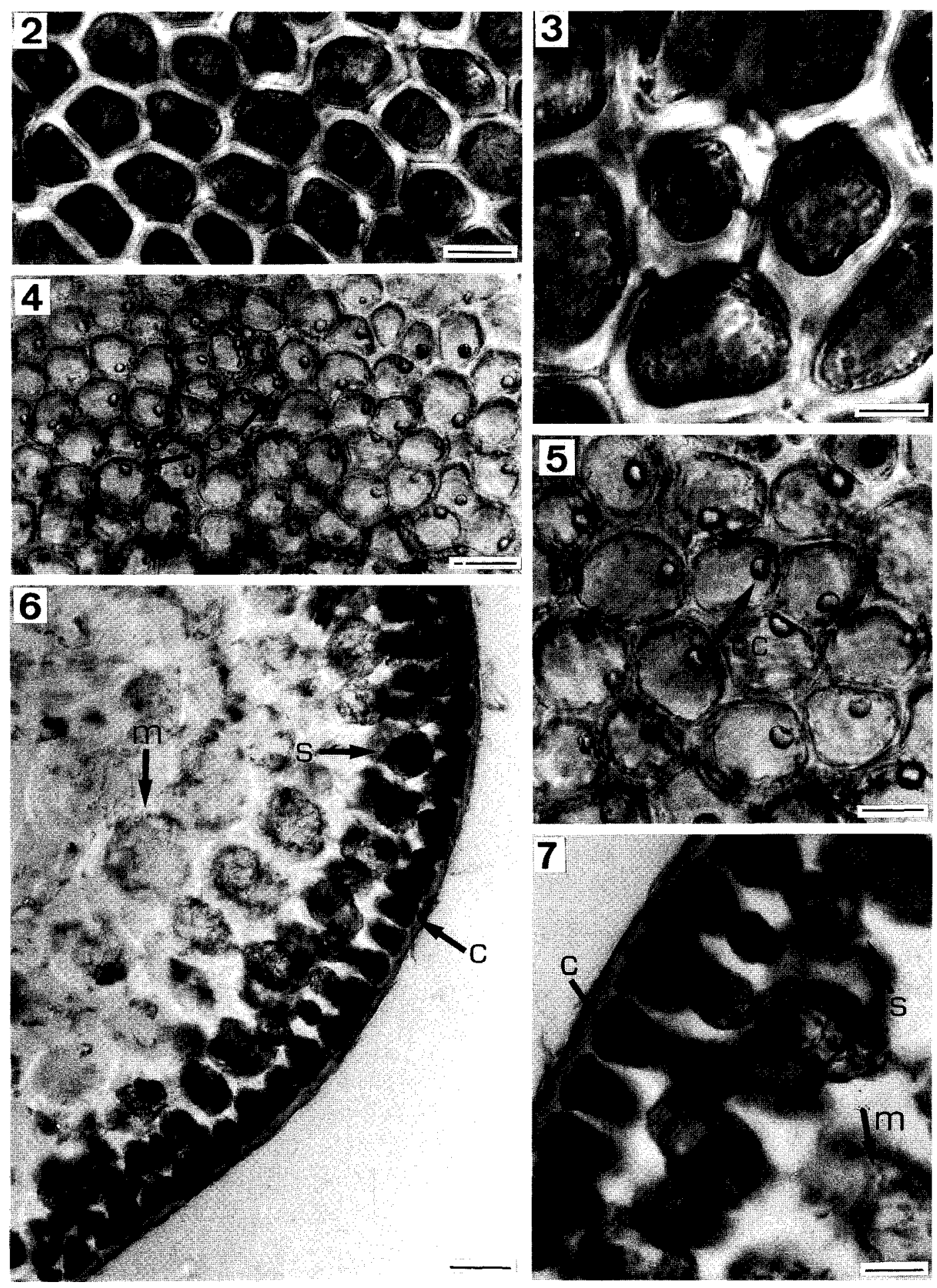

Figs 2-7. Laurencia viridis sp. nov.

Fig. 2. Surface view, formalin preserved plant (scale bar $=15 \mu \mathrm{m}$ ). Fig. 3. Surface cell, formalin preserved plant (scale bar $=8 \mu \mathrm{m}$ ). Fig. 4. Surface cell with single "corps à cérise", fresh plants (scale bar $=25 \mu \mathrm{m}$ ). Fig. 5. "corps à cérise" (scale bar $=18 \mu \mathrm{m})$. Figs $6-7$. Transverse sections, in the apical region of axes: cortical cells (c), subcortical cells $($ ) and medullary cells (m) (Fig. 6 scale bar $=35 \mu \mathrm{m}$ and Fig. 7 scale bar $=30 \mu \mathrm{m}$ ). 
rhizoids. The plants have a distinct pit enclosing several deciduous trichoflasts at the apex of each branch. The trichoblasts are subdichotomously branched to 5 or 6 orders. Each cell of the trichoblast possesses a single clear, refringent body ('corps à cérise').
Erect axes are $2-(4-8)-15 \mathrm{~cm}$ high and are $900-$ $(920-980)-1050 \mu \mathrm{m}$ in diameter basally, 1200$(1300-1400)-1500 \mu \mathrm{m}$ in median parts and $1100-$ $(1150-1180)-1250 \mu \mathrm{m}$ near the apex. Primary branches of the erect axes are $600-(830-920)-950$
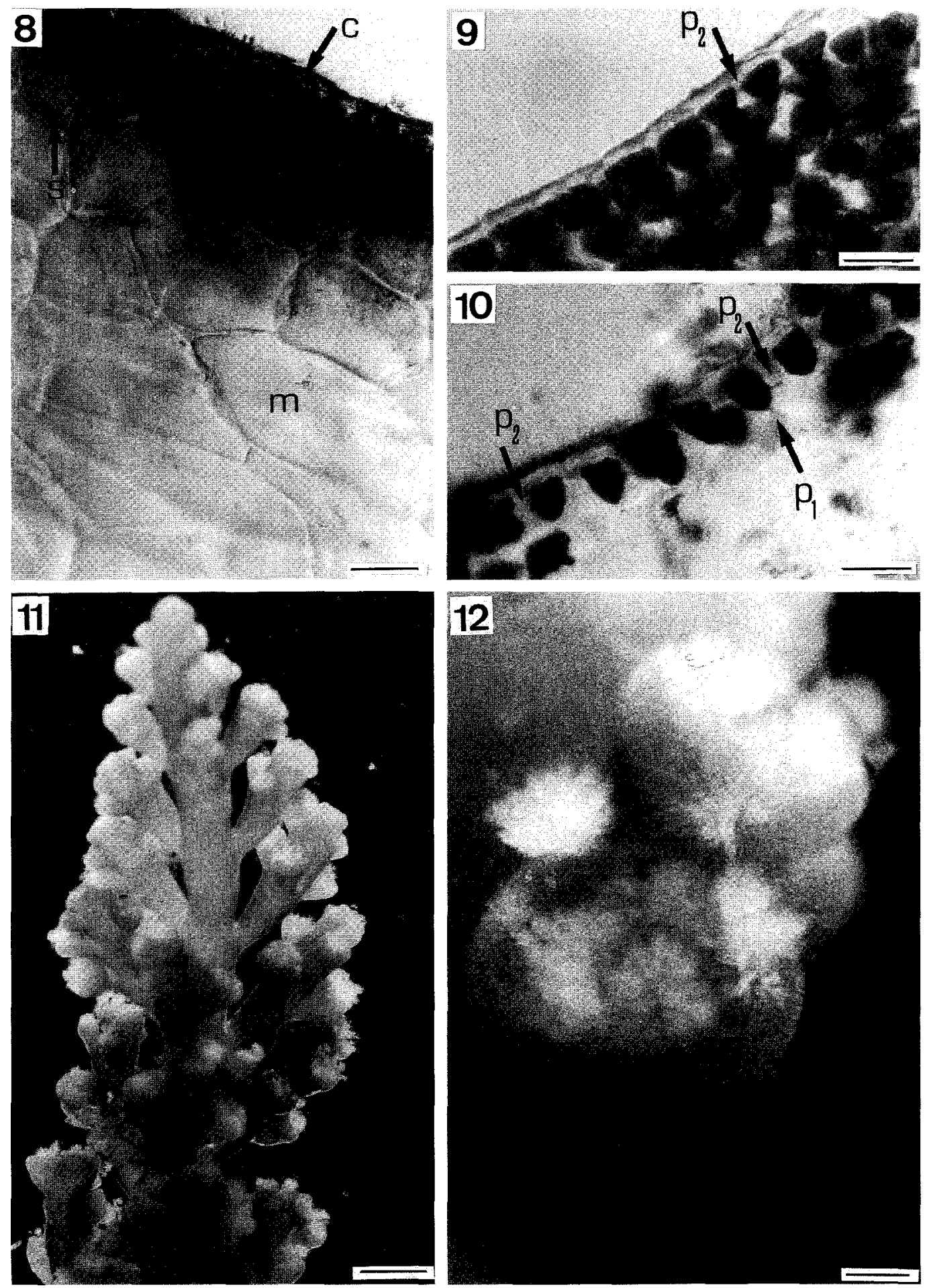

Figs 8-12. Laurencia viridis $s p$. nov.

Fig. 8. Longitudinal section of axis, cortical cells (c), subcortical cells (s) and medullary cells (m) (scale bar $=35 \mu \mathrm{m})$. Figs. $9-10$. Sections to show cortical cells connected longitudinally (p1) and laterally (p2) (scale bar $=30 \mu \mathrm{m}$ ). Fig. 11. Male gametophyte with spermatangial branches and sterile trichoblasts (scale bar $=0.25 \mathrm{~cm}$ ). Fig. 12. Detail of spermatangial cuplike branches (scale bar $=0.25 \mathrm{~mm}$ ). 
$\mu \mathrm{m}$ in diameter and secondary branches $(400-(526-$ $600)-800 \mu \mathrm{m}$ in diameter. In surface view the cells are polygonal, $15-20 \mu \mathrm{m}$ wide $\times 20-28 \mu \mathrm{m}$ high (Figs $2-3$ ). They are irregularly arranged and with a single 'corps à cérise', in fresh plants (Figs 4-5).

The surface cells are not protuberant. In transverse sections of the axis in the apical region the cortical cells are triangular or subquadrate (Figs 6-7), 12$(15-22)-30 \mu \mathrm{m}$ wide $\times 15-(17-25)-35 \mu \mathrm{m}$ high. Those in median parts of of the axis are $22-(25-$ $28)-30 \mu \mathrm{m}$ wide $\times 28-(30-33)-40 \mu \mathrm{m}$ high, while those in the basal area are $20-(23-27)-30 \mu \mathrm{m}$ wide $\times 27-(28-30)-37 \mu \mathrm{m}$ high. Subcortical cells are ovoid to sub-spherical (Figs $6-7), 25-(30-35)-40$ $\mu \mathrm{m}$ wide $\times 32-(35-45)-50 \mu \mathrm{m}$ high, with less pig-
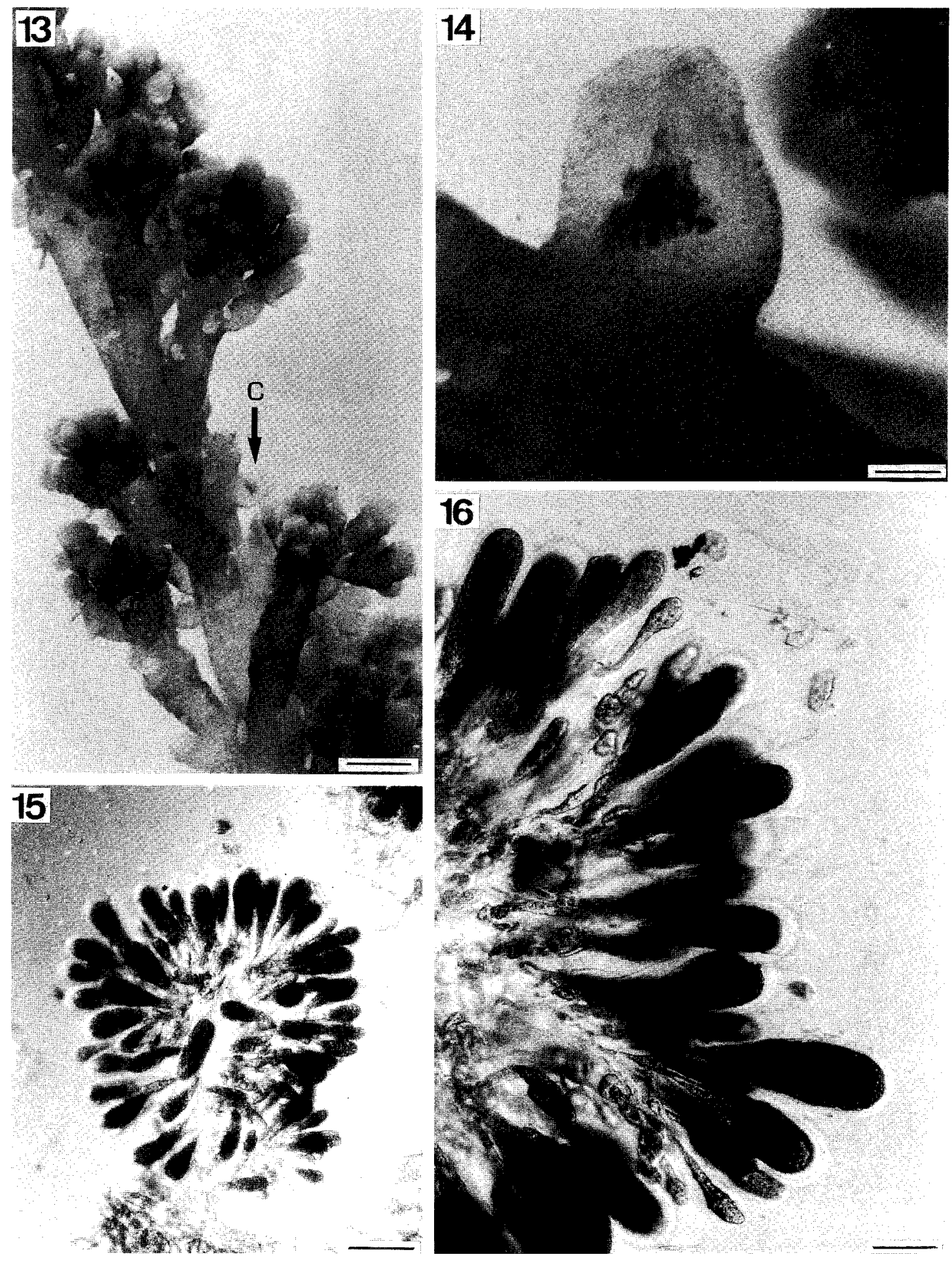

16

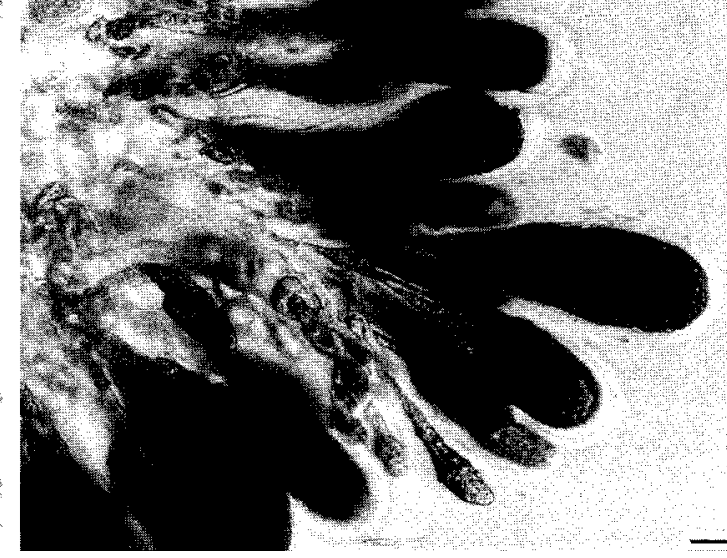

Figs 13-16. Laurencia viridis sp. nov.

Fig. 13. Female plants with cystocarp (c) $($ scale bar $=0.25 \mathrm{~cm}$ ). Fig. 14. Mature cystocarp (scale bar $=0.35 \mathrm{~mm}$ ). Figs $15-16$. Detail of carposporangia (Fig. 15 scale bar $=100 \mu \mathrm{m}$ and Fig. 16 scale bar $=25 \mu \mathrm{m}$ ). 
ment or even colourless. They lack lenticular thickenings and intercellular spaces. Medullary cells are large and colourless (Figs 6-7), 40-(50-60)-74 $\mu \mathrm{m}$ wide $\times 45-(50-70)-85 \mu \mathrm{m}$ high. In longitudinal section (Fig. 8), cortical cells are sub-ovate, $12-$ $(16-19)-25 \mu \mathrm{m}$ wide $\times 18-(20-22)-30 \mu \mathrm{m}$ high . Subcortical cells are ovate, $30-(35-48)-52 \mu \mathrm{m}$ wide $\times 40-(45-51)-54 \mu \mathrm{m}$ high. Medullary cells are very long, subrectangular, $60-(74-85)-90 \mu \mathrm{m}$ wide $\times 180-(210-250)-290 \mu \mathrm{m}$ high. Cortical cells are interconnected both longitudinally and laterally by secondary pit-connections (Figs 9-10). Subcortical and medullary cells show similar interconnections. Lenticular thickenings are absent.

\section{Reproductive structure}

Male gametophytes are $2-(3-4)-5 \mathrm{~cm}$ high (Fig. 11). Spermatangia are formed in dense clusters of repeatedly branched filaments arising from cup-like depressions (pits) at the apex of terminal branches (Fig. 12). Spermatangial branches are very small, $1-1,5 \mathrm{~mm}$ wide $\times 1,5-2-(3) \mathrm{mm}$ high. Spermatangial trichoblasts terminate in inflated, pyriform cells.
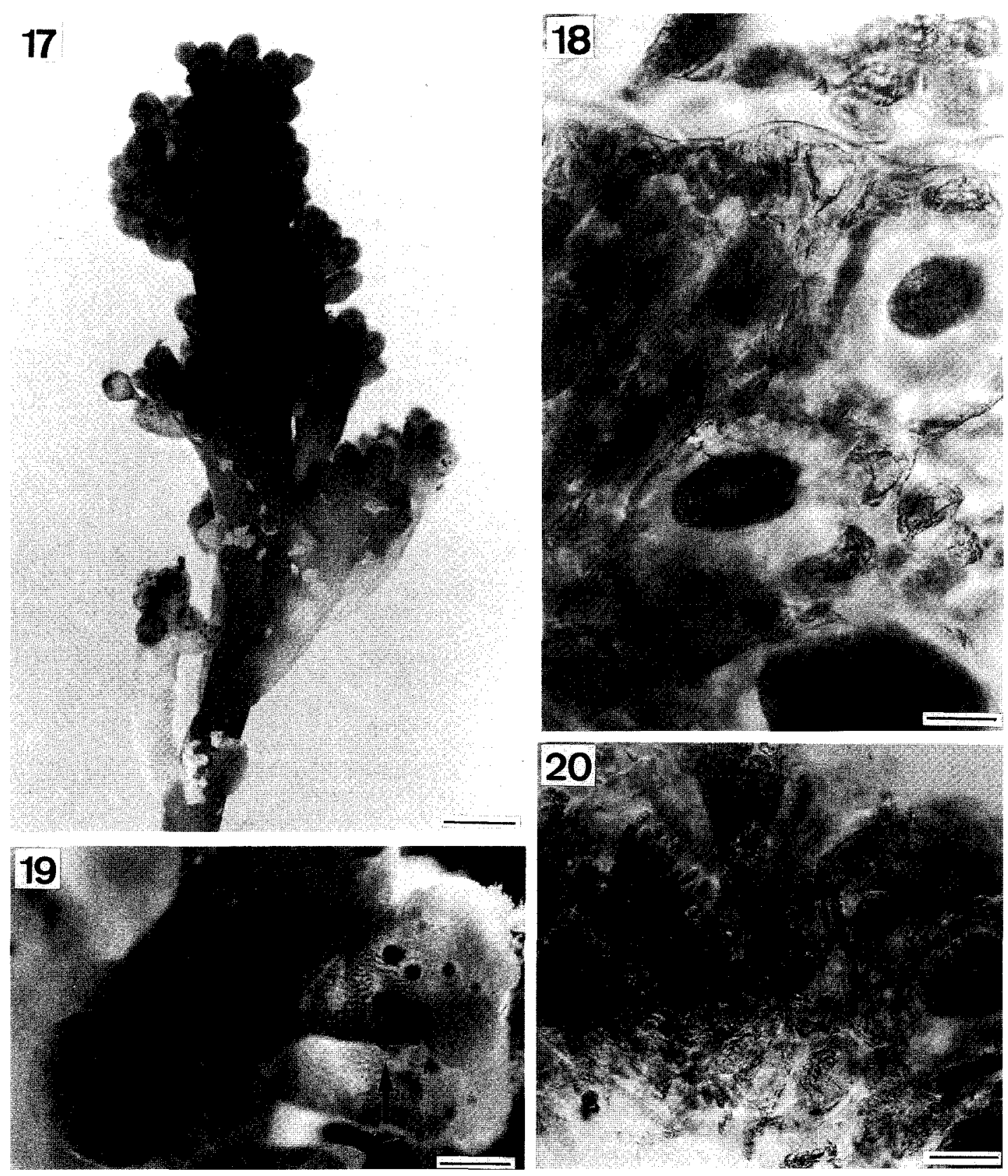

Figs 17-20. Laurencia viridis sp. nov.

Fig. 17. Tetrasporophytic plant (scale bar $=0.25 \mathrm{~cm}$ ). Fig. 18 and 20. Abaxial tetrasporangia (Fig. 18 scale bar $=60 \mu \mathrm{m}$ and Fig. 20 scale bar $=120 \mu \mathrm{m})$. Fig. 19. Tetrasporangia in parallel rows (scale bar $=0.35 \mathrm{~mm}$ ). 
Table I. Comparison of Laurencia spp from the Canaries which have been placed in the Subgenus Laurencia Saito

\begin{tabular}{|c|c|c|c|c|c|}
\hline Taxon & Habit & Main axes & $\begin{array}{l}\text { Cortical cells } \\
\text { (Tranv. Sect.) }\end{array}$ & $\begin{array}{l}\text { Lenticular } \\
\text { thickenings }\end{array}$ & Tetrasporangial \\
\hline L. obtusa & $\begin{array}{l}\text { - Dense tufts } \\
\text { intricate } \\
\text { with } \\
\text { stoloniferous } \\
\text { branches or } \\
\text { erect with } \\
\text { erect axes } \\
\text { from a } \\
\text { discoid } \\
\text { holfast. } \\
\text { - Pink-green. } \\
\text { - Irregular, } \\
\text { alternate, } \\
\text { opposite or } \\
\text { subvertici- } \\
\text { llate } \\
\text { branching } \\
\text { - } 2-5 \mathrm{~cm} \text { tall. }\end{array}$ & $\begin{aligned} \text { - } & \text { Terete. } \\
- & 0,5-0,9 \mathrm{~mm} \\
& \text { diam. }\end{aligned}$ & $\begin{array}{l}-2^{\circ} \text { pit- } \\
\text { connection } \\
\text { present. } \\
- \text { Non- } \\
\text { projecting. } \\
- \text { Isodiametric. } \\
-8-23 \mu \mathrm{m} \text { wide } \\
\times 15-25 \mu \mathrm{m} \\
\quad \text { high. } \\
- \text { 'Corps à } \\
\text { cérise' } 1-3 .\end{array}$ & - Absent. & $\begin{array}{l}\text { - Abaxial. } \\
\text { - Parallel. }\end{array}$ \\
\hline L. majuscula & $\begin{array}{l}\text { - Erect } \\
\text { thallus. } \\
\text { - Discoid } \\
\text { holdfast. } \\
\text { - Alternate or } \\
\text { opposite } \\
\text { branching. } \\
\text { - Axes to } 12 \mathrm{~cm} \\
\text { tall. }\end{array}$ & $\begin{array}{l}- \text { Terete. } \\
-1-2 \mathrm{~mm} . \\
\text { diam. }\end{array}$ & $\begin{array}{l}-2^{\circ} \text { pit- } \\
\text { connection } \\
\text { present. } \\
- \text { Projecting. } \\
- \text { Subquadrate. } \\
-20-35 \mu \mathrm{m} \text { wide } \\
\times 25-40 \mu \mathrm{m} \\
\text { high. } \\
- \text { 'Corps à } \\
\text { cérise' } 2-3 .\end{array}$ & - Absent. & $\begin{array}{l}\text { - Abaxial. } \\
\text { - Parallel. }\end{array}$ \\
\hline L. minuta & $\begin{array}{l}\text { - Erect thallus } \\
\text { very small. } \\
\text { - Pink or red. } \\
\text { - Discoid } \\
\text { holdfast, } \\
\text { stoloniferous } \\
\text { absent. } \\
-0,7-1 \mathrm{~cm} \\
\text { tall. }\end{array}$ & $\begin{aligned} \text { - } & \text { Terete. } \\
- & 0,3-0,7 \mathrm{~mm} \\
& \text { diam. }\end{aligned}$ & $\begin{array}{l}-2^{\circ} \text { pit- } \\
\text { connection } \\
\text { present. } \\
- \text { Projecting. } \\
- \text { Isodiametric. } \\
-8-10 \mu \mathrm{m} \text { wide } \\
\times 10-15 \mu \mathrm{m} \\
\text { high. } \\
- \text { 'Corps à } \\
\text { cérise' } 1\end{array}$ & - Absent. & $\begin{array}{l}\text { - Abaxial. } \\
\text { - Parallel. }\end{array}$ \\
\hline L. tenera & $\begin{array}{l}\text { - Colonies } \\
\text { intricate to } \\
5-10 \mathrm{~mm} \text { high. } \\
\text { - Brown-red and } \\
\text { yellow. } \\
\text { - Discoidal } \\
\text { haptera and } \\
\text { secondary } \\
\text { holdfast. } \\
\text { - Dichotomous } \\
\text { or } \\
\text { subdichoto- } \\
\text { mous } \\
\text { branching. } \\
-1 \mathrm{~cm} \text { tall. }\end{array}$ & $\begin{aligned} \text { - } & \text { Terete. } \\
- & 0,3-0,8 \mathrm{~mm} \\
& \text { diam. }\end{aligned}$ & $\begin{array}{l}-2^{\circ} \text { pit- } \\
\text { connection } \\
\text { present. } \\
- \text { Non- } \\
\text { projecting. } \\
- \text { Quadrate to } \\
\text { subquadrate. } \\
-18-22 \mu \mathrm{m} \text { wide } \\
\times 20-25 \mu \mathrm{m} \\
\text { high. } \\
- \text { 'Corps à } \\
\text { cérise' } \\
\text { absent. }\end{array}$ & - Absent. & $\begin{array}{l}\text { - Abaxial. } \\
\text { - Parallel. }\end{array}$ \\
\hline $\begin{array}{l}\text { L. viridis } \\
\text { sp. nov. }\end{array}$ & $\begin{array}{l}\text { - Erect } \\
\text { thallus. } \\
\text { - Bright green. } \\
\text { - Holdfast } \\
\text { stoloni- } \\
\text { ferous. } \\
\text { - Alternate } \\
\text { branching. } \\
\text { - Axes to } 15 \mathrm{~cm} \\
\text { tall. }\end{array}$ & $\begin{array}{l}\text { - Terete. } \\
-0,9-2 \mathrm{~mm} \\
\text { diam. }\end{array}$ & $\begin{array}{l}-2^{\circ} \text { pit- } \\
\text { connection } \\
\text { present. } \\
- \text { Non } \\
\text { projecting. } \\
- \text { Triangular o } \\
\text { subquadrate. } \\
-12-30 \mu \mathrm{m} \text { wide } \\
\times 15-40 \mu \mathrm{m} \\
\text { high. } \\
- \text { 'Corps à } \\
\text { cérise' } 1 .\end{array}$ & - Absent. & $\begin{array}{l}\text { - Abaxial. } \\
\text { - Parallel. }\end{array}$ \\
\hline
\end{tabular}


Table II. Comparison of Laurencia viridis with species of Laurencia with a green thallus from another parts of the world

\begin{tabular}{|c|c|c|c|c|c|}
\hline Taxon & Habit & Main axes & Cortical cells & $\begin{array}{l}\text { Lenticular } \\
\text { thickenings }\end{array}$ & Tetrasporangial \\
\hline $\begin{array}{l}\text { L. intricata } \\
\text { (Australia: } \\
\text { Cribb 1983) }\end{array}$ & $\begin{array}{l}\text { - Erect axes } \\
\text { densely } \\
\text { tufted } \\
\text { intricate. } \\
-10 \mathrm{~cm} \text { high. } \\
- \text { Stoloniferous } \\
\text { holdfast. } \\
- \text { Alternate } \\
\text { occasionally } \\
\text { subopposite. }\end{array}$ & $\begin{aligned}- & \text { Terete } \\
- & 0,7-1,1 \mathrm{~mm} \\
& \text { diam. }\end{aligned}$ & $\begin{array}{l}-2^{\circ} \text { pit- } \\
\text { connection } \\
\text { present. } \\
- \text { Occasionally } \\
\text { projecting. } \\
- \text { Non elongated } \\
\text { radially. } \\
- \text { Subquadrate. } \\
-2 \mu \mathrm{m} \text { diam. }\end{array}$ & - Absent & - Parallel \\
\hline $\begin{array}{l}\text { L. flexilis } \\
\text { (Australia: } \\
\text { Cribb 1983) }\end{array}$ & $\begin{array}{l}\text { - Erect axes. } \\
-4-5 \mathrm{~cm} \text { high. } \\
\text { - Stoloniferous } \\
\text { holdfast. } \\
\text { - Alternate, } \\
\text { irregulary } \\
\text { branching. }\end{array}$ & $\begin{array}{l}- \text { Terete } \\
-0,5-1 \mathrm{~mm} \\
\text { diam. }\end{array}$ & $\begin{aligned} &- 2^{\circ} \text { pit- } \\
& \text { connection } \\
& \text { absent. } \\
&- \text { Non } \\
& \text { projecting. } \\
&- \text { Elongated } \\
& \text { radially. } \\
&- \text { Subquadrate. } \\
&-17 \mu \mathrm{m} \text { diam. }\end{aligned}$ & - Absent & - Right-angle \\
\hline $\begin{array}{l}\text { L. nidifica } \\
\text { (Hawaii: } \\
\text { McDermid 1988) }\end{array}$ & $\begin{array}{l}\text { - Erect axes. } \\
-3-8 \mathrm{~cm} \text { high. } \\
\text { - Stoloniferous } \\
\text { holdfast. } \\
\text { - Alternate- } \\
\text { opposite } \\
\text { branching. }\end{array}$ & $\begin{array}{l}- \text { Terete } \\
-0,5-1 \mathrm{~mm} \\
\text { diam. }\end{array}$ & $\begin{array}{l}-2^{\circ} \text { pit- } \\
\text { connection } \\
\text { present. } \\
- \text { Non } \\
\text { projecting. } \\
- \text { Non elongated } \\
\text { radially. } \\
- \text { Subquadrate. } \\
-14 \mu \mathrm{m} \text { diam. }\end{array}$ & - Present & - Parallel \\
\hline $\begin{array}{l}\text { L. "green" } \\
\text { (Hawaii: } \\
\text { McDermid 1988) }\end{array}$ & $\begin{array}{l}\text { - Erect axes. } \\
-2-5 \mathrm{~cm} \text { high. } \\
- \text { Stoloniferous } \\
\text { holdfast. } \\
- \text { Alternate- } \\
\text { branching. }\end{array}$ & $\begin{array}{l}\text { - Terete } \\
-0,7-1 \mathrm{~mm} \\
\text { diam. }\end{array}$ & $\begin{aligned} &- 2^{\circ} \text { pit- } \\
& \text { connection } \\
& \text { present. } \\
&- \text { Non } \\
& \text { projecting. } \\
&- \text { Non elongated } \\
& \text { radially. } \\
&- \text { Subquadrate. } \\
&-22 \mu \mathrm{m} \text { diam. }\end{aligned}$ & - Absent & - Parallel \\
\hline $\begin{array}{l}\text { L. okamurai } \\
\text { (Japan: Saito } \\
\text { 1965; China: } \\
\text { Tseng 1983) }\end{array}$ & $\begin{array}{l}\text { - Erect axes } \\
\text { densely } \\
\text { tufted. } \\
\text { - } 4-19 \mathrm{~cm} \\
\text { high. } \\
\text { - Coalescing } \\
\text { basal } \\
\text { branches. } \\
\text { - Alternate, } \\
\text { opposite or } \\
\text { verticillate } \\
\text { branching. }\end{array}$ & $\begin{array}{l}- \text { Terete } \\
-0,8-1,2 \mathrm{~cm} \\
\text { diam. }\end{array}$ & $\begin{aligned} &- 2^{\circ} \text { pit- } \\
& \text { connection } \\
& \text { present. } \\
&- \text { Non } \\
& \text { projecting. } \\
&- \text { Non elongated } \\
& \text { radially. } \\
&- \text { Poligonal. } \\
&-14 \mu \mathrm{m} \text { long } \times \\
& 40 \mu \mathrm{m} \text { broad. }\end{aligned}$ & - Present & - Parallel \\
\hline $\begin{array}{l}\text { L. intermedia } \\
\text { (Caribbean: } \\
\text { Rodríguez-Rios } \\
\text { \& Saito 1982) }\end{array}$ & $\begin{array}{l}\text { - Erect axes, } \\
\text { loosely } \\
\text { tufted. } \\
-4-13 \mathrm{~cm} \\
\text { high. } \\
\text { - Stoloniferous } \\
\text { holdfast. } \\
\text { - Alternate } \\
\text { branching. }\end{array}$ & $\begin{array}{l}- \text { Terete } \\
-1-1,5 \mathrm{~mm} \\
\text { diam. }\end{array}$ & $\begin{aligned} &- 2^{\circ} \text { pit- } \\
& \text { connection } \\
& \text { absent. } \\
&- \text { Non } \\
& \text { projecting. } \\
& \text { - Elongated } \\
& \text { radially. } \\
&- \text { Poligonal. } \\
&-14 \mu \mathrm{m} \text { long } \times \\
& 17 \mu \mathrm{m} \text { broad. }\end{aligned}$ & - Absent & - Right-angle \\
\hline $\begin{array}{l}\text { L. viridis } \mathrm{sp} \text {. } \\
\text { nov. } \\
\text { (Macaronesian } \\
\text { Archipelago) }\end{array}$ & $\begin{aligned} \text { - Erect axes. } \\
-2-15 \mathrm{~cm} \\
\text { high. } \\
\text { - Stoloniferous } \\
\text { holdfast. } \\
\text { - Alternate } \\
\text { branching. }\end{aligned}$ & $\begin{array}{l}- \text { Terete } \\
-0,9-2 \mathrm{~mm} \\
\text { diam. }\end{array}$ & $\begin{array}{l}-2^{\circ} \text { pit- } \\
\text { connection } \\
\text { present. } \\
- \text { Non } \\
\text { projecting. } \\
- \text { Non elongated } \\
\text { radially. } \\
- \text { Triangular or } \\
\text { subquadrate. } \\
-25 \mu \mathrm{m} \text { long } \times \\
22 \mu \mathrm{m} \text { broad. }\end{array}$ & - Absent & - Parallel \\
\hline
\end{tabular}


Female plants are $4-(5-6)-10 \mathrm{~cm}$ high (Fig. 13). The mature carposporophyte is partially immersed in the thallus. Cystocarps are urceolate, sessile, projecting $1-1,5-(2) \mathrm{mm}$ above the thallus and $0,5-1-$ $(1,5) \mathrm{mm}$ in diameter (Fig. 14). Carposporangia are obovate, $15-35 \mu \mathrm{m}$ in wide and $100-150 \mu \mathrm{m}$ high (Figs 15-16). A delicate network of dichotomously branched filaments radiates from this complex of cells and lines the inner wall of the pericarp.

Tetrasporophytes are $3-(4-8)-15 \mathrm{~cm}$ high (Fig. 17). Tetrasporangia occur in parallel rows (Figs 17, 19) of 3-6 and are immersed at the tips of the terminal branches; the thallus continues to grow after tetrasporangial formation. Tetrasporangia are cut off abaxially (Figs 18, 20) from pericentral cells and cleave tetrahedrally. Mature tetrasporangia are $80-$ $110 \mu \mathrm{m}$ wide $\times 110-180 \mu \mathrm{m}$ high.

\section{Habitat}

Laurencia viridis grows in the lower intertidal zone, intermingled with other turf algae. It occurs on exposed, overhanging rocks subject to strong waveaction. Laurencia viridis is an annual plant that grows rapidly during winter-spring months and decays in late summer. This species would therefore have an ephemerophycean life-form (Garbary 1976).

Epiphytes: Erythrocystis, Janczewskia, Jania and Fosliella are very often observed along the axes of this species (Figs 13-16).

\section{Discussion and Conclusion}

Several morphological and anatomical characteristics of Laurencia viridis sp. nov., such as the branching pattern, general morphology and colour, are shared by previously described Laurencia species in the Canaries, which are compared in Table I.

In Table II we compare different green species of Laurencia from other parts of the world (Australia,
Hawaii, Japan, China and Caribbean) with $L$. viridis from the Macaronesian Archipelagos. We have considered the following characters: habit, main axes, cortical cells, lenticular thickening and tetrasporangials. The cortical cells (secondary pit-connections present or absent, non elongated radially or elongate radially), lenticular thickenings and tetrasporangials are the more important characters in which to see the differences between these species.

Laurencia viridis sp. nov. is a distinct species that lies within the generic criteria of Laurencia as described by Kylin (1956), Saito and Womersley (1974), and McDermid (1988). The general vegetative and reproductive features of the genus are described by Saito $(1967,1969)$. The presence of secondary pit-connections between adjacent cortical cells and the parallel arrangement of mature tetrasporangia place Laurencia viridis in the subgenus Laurencia (Saito 1967, 1969; Saito and Womersley 1974).

Although Laurencia has been the subject of several studies over recent years (Yamada 1931, Tseng 1943, Cribb 1958, 1983, Saito 1967, Saito and Womersley 1974), many researchers are of the opinion that systematics of the genus need further investigation (Saito 1982, McDermid 1989). Gil-Rodríguez and Haroun (1992) and Gil-Rodríguez (1992) have discussed the problems of subgeneric placement of several Laurencia spp. from the Canaries.

\section{Acknowledgements}

This work was supported by an investigation project of the Ministry of Education and Sciences of the Spanish Government (DGYCIT, PB 89-0601). We would like the thank Dr Y. Saito for his helpfull comment about the status of this species. We thank Dr W. F. Prud'homme van Reine for providing us with the CANCAP collections; Dr J. H. Price and Dr P. C. Silva for criticizing the manuscript; Dr José González Luis for the Latin translation and J. Reyes and M. C. Hernández for help with photography.

\section{References}

Cribb, A. B. 1958. Records of marine algae from southeastern Queensland-III. Laurencia Lamx. Univ. Queensland Papers, Dept. of Botany 3: 159-191.

Cribb, A. B. 1983. Marine Algae of the Southern Great Barrier Reef: Rhodophyta. Australian Coral Reef Society, Watson Ferguson and Co., Brisbane. 246. pp.

Garbary, D. 1976. Life-forms of algae and their distribution. Bot. Mar. 19: 97-106.

Gil-Rodríguez, M. C. and R. J. Haroun. 1992. The genus of Laurencia (Rhodomelaceae, Rhodophyta) in the Canary Islands. Cour. Forsch. Inst. Senckenberg.

Gil-Rodríguez, M. C. 1992. Metodología para la comprobación experimental de la existencia de "morfotipos" en algas rojas. (Conferencia en el IX Simposio Nacional de Botánica Criptogámica. Salamanca 1991).

Kylin, H. 1956. Die Gattungen der Rhodophyceen. C. W. K. Gleerup, Lund. 673 pp.

McDermid, K. J. 1988 a. Section V. Laurencia (Rhodophyta, Rhodomelaceae). Introduction. In: (I. A. Abbott, ed.) Taxonomy of Economic Seaweeds with Reference to some Pacific and Caribbean species Vol. II. Caliornia Sea Grant College Program, La Jolla. pp. $221-229$. 
McDermid, K. J. 1988 b. Laurencia from Hawai Islands: Key, annotated list, and distribution of the species. In: (I. A. Abbott, ed.) Taxonomy of Economic Seaweeds with Reference to some Pacific and Caribbean species (Vol. II. California Sea Grant College Program, La Jolla. pp. 231-247.

McDermid, K. J. 1989. Laurencia crustiformans sp. nov. (Ceramiales, Rhodophyta) from the Hawaiian Islands. Phycologia 28: $352-259$.

Rodríguez de Rios, N. and Y. Saito. 1982. Observaciones sobre el género Laurencia en Venezuela. I: Laurencia intermedia Yamada y Laurencia corallopsis (Montagne) Howe. Ernstia 11: $1-16$.

Saito, Y. 1965. Contributions to the morphology of the genus Laurencia of Japan. II. Bull. Fac. Fish., Hokkaido Univ. 15(4): $207-212$.

Saito, Y. 1967. Studies on Japanese species of Laurencia with special reference to their comparative morphology. Mem. Fac. Fish. Hokkaido Univ. 15: 1-81.

Saito, Y. 1969. The algal genus Laurencia from the Hawaiian Islands, the Philippine Islands and adjacent areas. Pac. Sci. 23: $148-160$.
Saito, Y. 1982. Morphology and infrageneric position of three British species of Laurencia (Ceramiales, Rhodophyta). Phycologia 21: 299-306.

Saito, Y. and H. B. S. Womersley. 1974. The southern Australian species of Laurencia (Ceramiales: Rhodophyta). Aust. J. Bot. 22: 815-874.

Tseng, C. K. 1943. Marine algae of Hong Kong. IV. The genus Laurencia. Pap. Mich. Acad. Sci. 28: 185-208. .

Tseng, C. K. 1983. Common Seaweeds of China. Institute of Oceanology, Academia Sinica, Quigdao, China. Science Press, Beijing, China.

Vandermeulen, H., D. J. Garbary and M. D. Guiry. 1990. Laurencia minuta sp. nov. (Ceramiales, Rhodomelaceae), a diminutive red alga from the Gulf of Aqaba (Red Sea). Br. phycol. J. 25: 237-244.

Womersley, H. B. S. 1984. The Marine Benthic Flora of Southern Australia. Part I, Government Printer, South Australia. $329 \mathrm{pp}$.

Yamada, Y. 1931. Notes on Laurencia, with special reference to the Japanese species. Univ. Calif. Publs Bot. 16: 185310. 